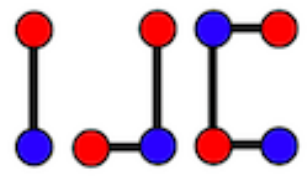

\title{
Computing the split domination number of grid graphs
}

\author{
V. R. Girish ${ }^{\mathrm{a}}$, P. Usha ${ }^{\mathrm{b}}$ \\ ${ }^{a}$ Department of Science and Humanities, PES University(EC Campus), \\ Electronic City, Bengaluru, Karnataka, India \\ ${ }^{b}$ Department of Mathematics, Siddaganga Institue of Technology, Tumakuru, Karnataka, India \\ girishvr1@pes.edu, ushapmurhty@gmail.com
}

\begin{abstract}
A set $D \subseteq V$ is a dominating set of $G$ if every vertex in $V-D$ is adjacent to some vertex in $D$. The dominating number $\gamma(G)$ of $G$ is the minimum cardinality of a dominating set $D$. A dominating set $D$ of a graph $G=(V, E)$ is a split dominating set if the induced graph $\langle V-D\rangle$ is disconnected. The split domination number $\gamma_{s}(G)$ is the minimum cardinality of a split domination set. In this paper we have introduced a new method to obtain the split domination number of grid graphs by partitioning the vertex set in terms of star graphs and also we have obtained the exact values of $\gamma_{s}\left(G_{m, n}\right), m \leq n, m, n \leq 24$.
\end{abstract}

Keywords: separate, domination number, split domination, grid graph, star graph

Mathematics Subject Classification: 05C69

DOI: $10.19184 / \mathrm{ijc} .2021 .5 .1 .1$

\section{Introduction}

The graphs considered here are finite, connected, undirected without loops or multiple edges and without isolated vertices. As usual $n$ and $q$ denote the number of vertices and edges of a graph $G$. For any undefined term or notation in this paper can be found in Harary [2].

A set $D \subseteq V$ is a dominating set of $G$ if every vertex in $V-D$ is adjacent to some vertex in $D$. The dominating number $\gamma(G)$ of $G$ is the minimum cardinality of a dominating set $D$ [5]. V.R.

Received: 19 September 2018, Revised: 13 December 2020, Accepted: 15 March 2021. 
Kulli and B. Janakiram had introduced a concept of split domination [3]. A dominating set D of a graph $G=(V, E)$ is a split dominating set, if the induced graph $\langle V-D\rangle$ is disconnected. The split domination number $\gamma_{s}(G)$ is the minimum cardinality of a split domination set.

A two dimensional grid graph $G_{m, n}$ is the graph Cartesian product $P_{m} \times P_{n}$ of paths on $m$ and $n$ vertices. The Cartesian graph product of $G_{1} \times G_{2}$ with disjoint vertex sets and edge sets in $G_{1}, G_{2}$ is the graph with the vertex set $V_{1} \times V_{2}$ and two vertices $u=\left(u_{1}, u_{2}\right)$ and $v=\left(v_{1}, v_{2}\right)$ are adjacent in $G_{1} \times G_{2}$ whenever $\left[u_{1}=v_{1}\right.$ and $u_{2}$ adj $\left.v_{2}\right]$ or $\left[u_{2}=v_{2}\right.$ and $u_{1}$ adj $\left.v_{1}\right]$. A star graph is a complete bipartite graph of the form $K_{1, n-1}$ with $n$ vertices. The neighborhood of a vertex in the graph $G$ is the set of vertices adjacent to $v$ and is denoted by $N(v)$.

Computing of domination of grid graph has been studied in $[1,4]$. In this paper we have introduced a new method to obtain the split domination number of a grid graphs by partitioning the vertex set in terms of $K_{1,3}, K_{1,2}, K_{2}$ and $K_{1}$ and also we have obtained the exact values of $\gamma_{s}\left(G_{m, n}\right), m \leq n, m, n \leq 24$.

\section{Preliminaries}

To simplify the description of the algorithm, we first define an order of the vertices of an $m n$ grid graph with vertices $v_{i, j}, 1 \leq i \leq m, 1 \leq j \leq n$. Every minimum dominating set can be constructed by an exhaustive search where in each step any undominated vertex is picked, after which all possible ways of dominating this vertex are considered in turn.

The procedure to construct the minimum split dominating set is as follows:

Pick the vertex of degree 3 say $v_{i}, E=v_{i} \cup N\left(v_{i}\right)$. Next choose the vertex in $V(G)-E$, that has the vertex of degree 3 , if not choose the vertex of degree 2 otherwise choose the vertex of degree 1 say $v_{j}$, if not choose $v_{k} \in E$. Let $D=v_{i} \cup v_{j} \cup v_{k}$ such that the number of vertices in $D$ is minimum and $\langle V(G)-D\rangle$ is disconnected, this procedure is continued unless $V(G)-E$ is an empty set. Suppose if $\langle V(G)-D>$ is connected, then we need one more vertex to make the graph disconnected.

\section{Algorithm to Find the Split Domination Number of grid graph by partitioning the vertex} set.

Step 1: Divide the grid graph $G_{m, n}$ by partitioning the vertex set in terms $K_{1,3}, K_{1,2}, K_{1,1}$ and $K_{1}$ such that

(i) Partitioning $A=\left\{P_{1}, P_{2}, P_{3} \ldots . . P_{n}\right\}$ is minimum.

(ii) $<V\left(P_{1}\right)>\cap<V\left(P_{2}\right)>\cap \ldots \ldots \ldots \cap<V\left(P_{n}\right)>=\phi$ and $v_{1} \in<V\left(P_{1}\right)>$, $v_{2} \in<V\left(P_{2}\right)>$ if $\operatorname{deg}\left(v_{1}\right)$ is maximum in $\left\langle V\left(P_{1}\right)>\right.$ and $v_{2} \notin N\left(v_{1}\right)$ in $G_{m, n}$.

(iii) $E\left(P_{1}\right) \cap E\left(P_{2}\right)=\phi, E\left(P_{1}\right)$ is the edges in $\left\langle V\left(P_{1}\right)\right\rangle$.

Step 2: Suppose $A$ contains atleast two partition set say $P_{1}, P_{2}$ such that $\left\langle V\left(P_{1}\right)\right\rangle=K_{1}$ and $<V\left(P_{2}\right)>=K_{1}$ and $H=\left\{v_{c} \in N\left(V\left(P_{1}\right) \cap V\left(P_{2}\right)\right)\right.$ in $\left.G_{m, n}\right\}, A=\left\{A-\left\{P_{1}, P_{2}\right\}\right\}$.

Step 3: Let $D=\left\{v_{i} \in<V\left(P_{i}\right)>, P_{i} \in A / v_{i}\right.$ is of max deg of $<V\left(P_{i}\right)>$ and $<V\left(P_{i}\right)>\neq$ $\left.K_{1,1}\right\}$ 
Step 4: IF $A$ contains atleast one partition $P_{j}$, such that $\left\langle V\left(P_{j}\right)>=K_{1,1}\right.$ with $\left\langle V\left(P_{j}\right)\right\rangle=$ $\left\{v_{m}, v_{r}\right\}$.

GOTO STEP 5.

ELSE IF $H=\phi$

$C=D$

ELSE

$C=D \cup H$

GOTO STEP 7.

Step 5: For each partition $P_{j}$.

IF atleast one vertex say $v_{s}, v_{s} \in N\left(v_{m}\right), v_{s} \notin<V\left(P_{j}\right)>$ and $N\left(v_{s}\right)-\left\{v_{m}\right\} \in D$.

$\left\{v_{m}\right\} \in S$.

ELSE

$\left\{v_{m}\right\}$ or $\left\{v_{r}\right\} \in S$.

GOTO STEP 5.

Let $S$ be the set of all such vertices.

Step 6: IF $H=\phi$ and $S=\phi$

$C=D$

ELSE IF $H=\phi$ and $S \neq \phi$

$C=D \cup S$.

ELSE

$C=D \cup S \cup H$.

Step 7: Find the split adjacency matrix

$$
a_{i j}= \begin{cases}v_{i j}=1 & v_{i} \text { is adjacent to } v_{j} \text { and } v_{i} \notin C \\ v_{i j}=0 & \text { Otherwise. }\end{cases}
$$

Step 8: IF $a_{i j}$ contains atleast one zero row then, $\gamma_{s}=|C|$

GOTO STEP 11.

ELSE

GOTO STEP 9.

Step 9: Let $\left\{v_{k}\right\}$ is the row in $a_{i j}$ in which sum of all the elements in $\left\{v_{k}\right\}=1$ and 1 is present in $v_{p}$ column.

Step 10: $\gamma_{s}=|C|+\left|\left\{v_{p}\right\}\right|=|C|+1$.

Step 11: END

\section{Examples}

$$
\begin{aligned}
& A=\left\{P_{1}, P_{2}, P_{3}, P_{4}, P_{6}\right\} \\
& D=\left\{v_{2}, v_{11}, v_{9}, v_{18}, v_{5}\right\}
\end{aligned}
$$




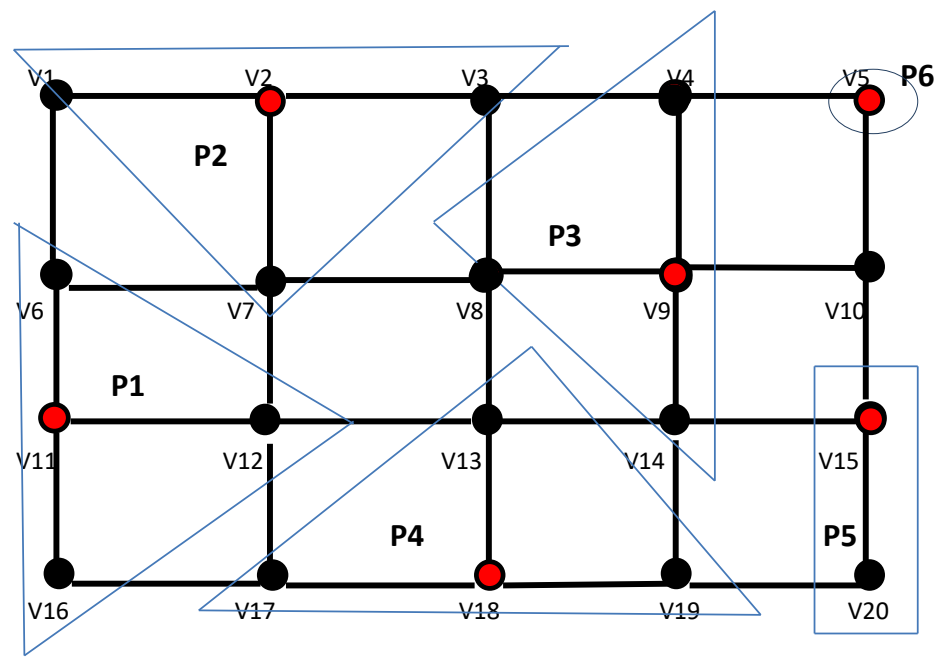

Figure 1. A grid graph $G_{4,5}$

For partition $P_{5}, v_{s}=v_{10}, v_{m}=15, S=\left\{v_{15}\right\}$

$$
C=D \cup S=\left\{v_{2}, v_{11}, v_{9}, v_{18}, v_{5}, v_{15}\right\}
$$

The Split adjacent matrix $a_{i j}$ is:

\begin{tabular}{|c|c|c|c|c|c|c|c|c|c|c|c|c|c|c|c|c|c|c|c|}
\hline$v_{1}$ & $v_{2}$ & $v_{3}$ & $v_{4}$ & $v_{5}$ & $v_{6}$ & $v_{7}$ & $v_{8}$ & $v_{9}$ & $v_{10}$ & $v_{11}$ & $v_{12}$ & $v_{13}$ & $v_{14}$ & $v_{15}$ & $v_{16}$ & $v_{17}$ & $v_{18}$ & $v_{19}$ & $v_{20}$ \\
\hline$(0$ & 0 & 0 & 0 & 0 & 1 & 0 & 0 & 0 & 0 & 0 & 0 & 0 & 0 & 0 & 0 & 0 & 0 & 0 & 0 \\
\hline 1 & 0 & 1 & 0 & 0 & 0 & 1 & 0 & 0 & 0 & 0 & 0 & 0 & 0 & 0 & 0 & 0 & 0 & 0 & 0 \\
\hline 0 & 0 & 0 & 1 & 0 & 0 & 0 & 1 & 0 & 0 & 0 & 0 & 0 & 0 & 0 & 0 & 0 & 0 & 0 & 0 \\
\hline 0 & 0 & 1 & 0 & 0 & 0 & 0 & 0 & 0 & 0 & 0 & 0 & 0 & 0 & 0 & 0 & 0 & 0 & 0 & 0 \\
\hline 0 & 0 & 0 & 1 & 0 & 0 & 0 & 0 & 0 & 1 & 0 & 0 & 0 & 0 & 0 & 0 & 0 & 0 & 0 & 0 \\
\hline 1 & 0 & 0 & 0 & 0 & 0 & 1 & 0 & 0 & 0 & 0 & 0 & 0 & 0 & 0 & 0 & 0 & 0 & 0 & 0 \\
\hline 0 & 0 & 0 & 0 & 0 & 1 & 0 & 1 & 0 & 0 & 0 & 1 & 0 & 0 & 0 & 0 & 0 & 0 & 0 & 0 \\
\hline 0 & 0 & 1 & 0 & 0 & 0 & 1 & 0 & 0 & 0 & 0 & 0 & 1 & 0 & 0 & 0 & 0 & 0 & 0 & 0 \\
\hline 0 & 0 & 0 & 1 & 0 & 0 & 0 & 1 & 0 & 1 & 0 & 0 & 0 & 1 & 0 & 0 & 0 & 0 & 0 & 0 \\
\hline 0 & 0 & 0 & 0 & 0 & 0 & 0 & 0 & 0 & 0 & 0 & 0 & 0 & 0 & 0 & 0 & 0 & 0 & 0 & 0 \\
\hline 0 & 0 & 0 & 0 & 0 & 1 & 0 & 0 & 0 & 0 & 0 & 1 & 0 & 0 & 0 & 1 & 0 & 0 & 0 & 0 \\
\hline 0 & 0 & 0 & 0 & 0 & 0 & 1 & 0 & 0 & 0 & 0 & 0 & 1 & 0 & 0 & 0 & 1 & 0 & 0 & 0 \\
\hline 0 & 0 & 0 & 0 & 0 & 0 & 0 & 1 & 0 & 0 & 0 & 1 & 0 & 1 & 0 & 0 & 0 & 0 & 0 & 0 \\
\hline 0 & 0 & 0 & 0 & 0 & 0 & 0 & 0 & 0 & 0 & 0 & 0 & 1 & 0 & 0 & 0 & 0 & 0 & 1 & 0 \\
\hline 0 & 0 & 0 & 0 & 0 & 0 & 0 & 0 & 0 & 1 & 0 & 0 & 0 & 1 & 0 & 0 & 0 & 0 & 0 & 1 \\
\hline 0 & 0 & 0 & 0 & 0 & 0 & 0 & 0 & 0 & 0 & 0 & 0 & 0 & 0 & 0 & 0 & 1 & 0 & 0 & 0 \\
\hline 0 & 0 & 0 & 0 & 0 & 0 & 0 & 0 & 0 & 0 & 0 & 1 & 0 & 0 & 0 & 1 & 0 & 0 & 0 & 0 \\
\hline 0 & 0 & 0 & 0 & 0 & 0 & 0 & 0 & 0 & 0 & 0 & 0 & 1 & 0 & 0 & 0 & 1 & 0 & 1 & 0 \\
\hline 0 & 0 & 0 & 0 & 0 & 0 & 0 & 0 & 0 & 0 & 0 & 0 & 0 & 1 & 0 & 0 & 0 & 0 & 0 & 1 \\
\hline $20 \backslash 0$ & 0 & 0 & 0 & 0 & 0 & 0 & 0 & 0 & 0 & 0 & 0 & 0 & 0 & 0 & 0 & 0 & 0 & 1 & 0 \\
\hline
\end{tabular}

Since there exists a zero row $v_{10}$ in $a_{i j}$. therefore the split domination number $\gamma_{s}=|C|=6$. 


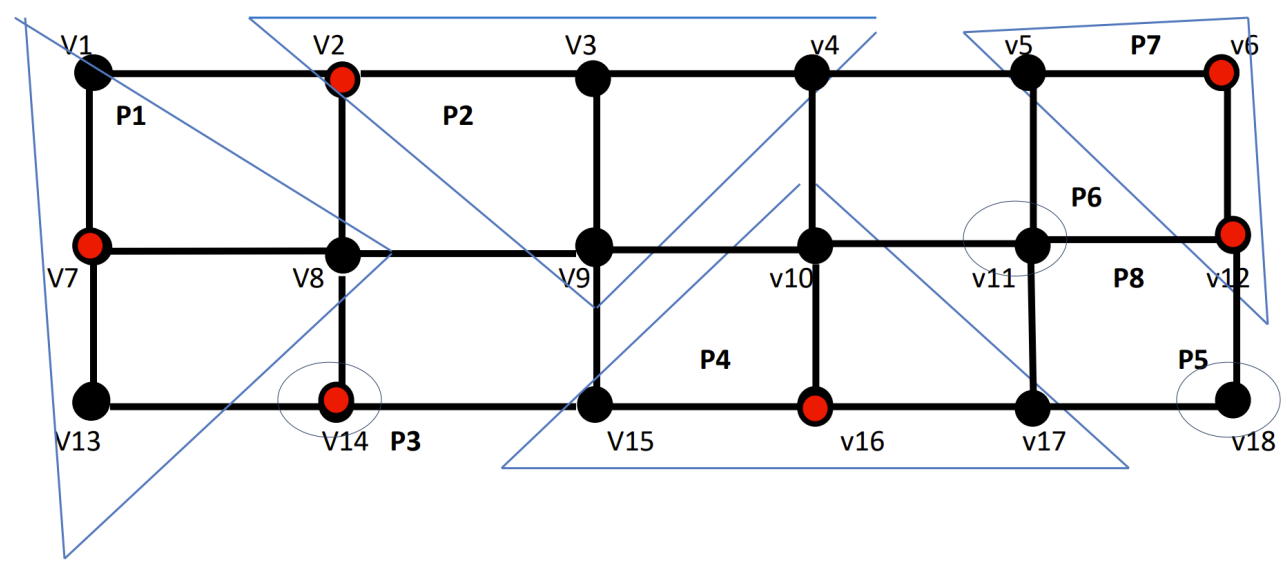

Figure 2. A grid graph $G_{3,6}$

$$
\begin{gathered}
A=\left\{P_{1}, P_{2}, P_{3}, P_{4}, P_{5}, P_{6}, P_{7}\right\} \\
\text { Since }<V\left(P_{5}\right)>\text { and }<V\left(P_{6}\right)>=K_{1} \text { and } H=\left\{v_{c}=v_{12}\right\} \\
A=\left\{P_{1}, P_{2}, P_{3}, P_{4}, P_{7}\right\} \\
D=\left\{v_{3}, v_{6}, v_{7}, v_{14}, v_{16}\right\} \\
C=D \cup H=\left\{v_{3}, v_{6}, v_{7}, v_{14}, v_{16}, v_{12}\right\}
\end{gathered}
$$

\begin{tabular}{|c|c|c|c|c|c|c|c|c|c|c|c|c|c|c|c|c|c|c|}
\hline & $v_{1}$ & $v_{2}$ & $v_{3}$ & $v_{4}$ & $v_{5}$ & $v_{6}$ & $v_{7}$ & $v_{8}$ & $v_{9}$ & $v_{10}$ & $v_{11}$ & $v_{12}$ & $v_{13}$ & $v_{14}$ & $v_{15}$ & $v_{16}$ & $v_{17}$ & $v_{18}$ \\
\hline$v_{1}$ & 0 & 1 & 0 & 0 & 0 & 0 & 0 & 0 & 0 & 0 & 0 & 0 & 0 & 0 & 0 & 0 & 0 & 0 \\
\hline$v_{2}$ & 1 & 0 & 0 & 0 & 0 & 0 & 0 & 1 & 0 & 0 & 0 & 0 & 0 & 0 & 0 & 0 & 0 & 0 \\
\hline$v_{3}$ & 0 & 1 & 0 & 1 & 0 & 0 & 0 & 0 & 1 & 0 & 0 & 0 & 0 & 0 & 0 & 0 & 0 & 0 \\
\hline$v_{4}$ & 0 & 0 & 0 & 0 & 1 & 0 & 0 & 0 & 0 & 1 & 0 & 0 & 0 & 0 & 0 & 0 & 0 & 0 \\
\hline$v_{5}$ & 0 & 0 & 0 & 1 & 0 & 0 & 0 & 0 & 0 & 0 & 1 & 0 & 0 & 0 & 0 & 0 & 0 & 0 \\
\hline$v_{6}$ & 0 & 0 & 0 & 0 & 1 & 0 & 0 & 0 & 0 & 0 & 0 & 0 & 0 & 0 & 0 & 0 & 0 & 0 \\
\hline$v_{7}$ & 1 & 0 & 0 & 0 & 0 & 0 & 0 & 1 & 0 & 0 & 0 & 0 & 1 & 0 & 0 & 0 & 0 & 0 \\
\hline$v_{8}$ & 0 & 1 & 0 & 0 & 0 & 0 & 0 & 0 & 1 & 0 & 0 & 0 & 0 & 0 & 0 & 0 & 0 & 0 \\
\hline$v_{9}$ & 0 & 0 & 0 & 0 & 0 & 0 & 0 & 1 & 0 & 1 & 0 & 0 & 0 & 0 & 1 & 0 & 0 & 0 \\
\hline$v_{10}$ & 0 & 0 & 0 & 1 & 0 & 0 & 0 & 0 & 1 & 0 & 1 & 0 & 0 & 0 & 0 & 0 & 0 & 0 \\
\hline$v_{11}$ & 0 & 0 & 0 & 0 & 1 & 0 & 0 & 0 & 0 & 1 & 0 & 0 & 0 & 0 & 0 & 0 & 1 & 0 \\
\hline$v_{12}$ & 0 & 0 & 0 & 0 & 0 & 0 & 0 & 0 & 0 & 0 & 1 & 0 & 0 & 0 & 0 & 0 & 0 & 1 \\
\hline$v_{13}$ & 0 & 0 & 0 & 0 & 0 & 0 & 0 & 0 & 0 & 0 & 0 & 0 & 0 & 0 & 0 & 0 & 0 & 0 \\
\hline$v_{14}$ & 0 & 0 & 0 & 0 & 0 & 0 & 0 & 1 & 0 & 0 & 0 & 0 & 1 & 0 & 1 & 0 & 0 & 0 \\
\hline$v_{15}$ & 0 & 0 & 0 & 0 & 0 & 0 & 0 & 0 & 1 & 0 & 0 & 0 & 0 & 0 & 0 & 0 & 0 & 0 \\
\hline$v_{16}$ & 0 & 0 & 0 & 0 & 0 & 0 & 0 & 0 & 0 & 1 & 0 & 0 & 0 & 0 & 1 & 0 & 1 & 0 \\
\hline$v_{17}$ & 0 & 0 & 0 & 0 & 0 & 0 & 0 & 0 & 0 & 0 & 1 & 0 & 0 & 0 & 0 & 0 & 0 & 1 \\
\hline$v_{18}$ & 10 & 0 & 0 & 0 & 0 & 0 & 0 & 0 & 0 & 0 & 0 & 0 & 0 & 0 & 0 & 0 & 1 & 0 \\
\hline
\end{tabular}

The Split adjacent matrix $a_{i j}$ is: 
Since there exists a zero row $v_{13}$ in $a_{i j}, \gamma_{s}=|C|=6$

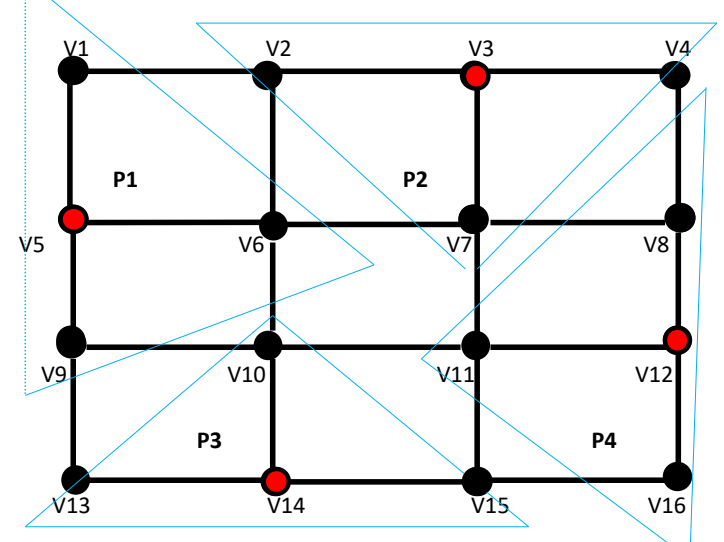

Figure 3. A grid graph $G_{4,4}$

$$
\begin{gathered}
A=\left\{P_{1}, P_{2}, P_{3}, P_{4}\right\} \\
D=\left\{v_{3}, v_{5}, v_{12}, v_{14}\right\} \\
H=\phi \\
C=D=\left\{v_{3}, v_{5}, v_{12}, v_{14}\right\}
\end{gathered}
$$

\begin{tabular}{|c|c|c|c|c|c|c|c|c|c|c|c|c|c|c|c|c|}
\hline & $v_{1}$ & $v_{2}$ & $v_{3}$ & $v_{4}$ & $v_{5}$ & $v_{6}$ & $v_{7}$ & $v_{8}$ & $v_{9}$ & $v_{10}$ & $v_{11}$ & $v_{12}$ & $v_{13}$ & $v_{14}$ & $v_{15}$ & $v_{16}$ \\
\hline$v_{1}$ & 0 & 1 & 0 & 0 & 0 & 0 & 0 & 0 & 0 & 0 & 0 & 0 & 0 & 0 & 0 & 0 \\
\hline$v_{2}$ & 1 & 0 & 0 & 0 & 0 & 1 & 0 & 0 & 0 & 0 & 0 & 0 & 0 & 0 & 0 & 0 \\
\hline$v_{3}$ & 0 & 1 & 0 & 1 & 0 & 0 & 1 & 0 & 0 & 0 & 0 & 0 & 0 & 0 & 0 & 0 \\
\hline$v_{4}$ & 0 & 0 & 0 & 0 & 0 & 0 & 0 & 1 & 0 & 0 & 0 & 0 & 0 & 0 & 0 & 0 \\
\hline$v_{5}$ & 1 & 0 & 0 & 0 & 0 & 1 & 0 & 0 & 1 & 0 & 0 & 0 & 0 & 0 & 0 & 0 \\
\hline$v_{6}$ & 0 & 1 & 0 & 0 & 0 & 0 & 1 & 0 & 0 & 1 & 0 & 0 & 0 & 0 & 0 & 0 \\
\hline$v_{7}$ & 0 & 0 & 0 & 0 & 0 & 1 & 0 & 1 & 0 & 0 & 1 & 0 & 0 & 0 & 0 & 0 \\
\hline$v_{8}$ & 0 & 0 & 0 & 1 & 0 & 0 & 1 & 0 & 0 & 0 & 0 & 0 & 0 & 0 & 0 & 0 \\
\hline$v_{9}$ & 0 & 0 & 0 & 0 & 0 & 0 & 0 & 0 & 0 & 1 & 0 & 0 & 1 & 0 & 0 & 0 \\
\hline$v_{10}$ & 0 & 0 & 0 & 0 & 0 & 1 & 0 & 0 & 1 & 0 & 1 & 0 & 0 & 0 & 0 & 0 \\
\hline$v_{11}$ & 0 & 0 & 0 & 0 & 0 & 0 & 1 & 0 & 0 & 1 & 0 & 0 & 0 & 0 & 1 & 0 \\
\hline$v_{12}$ & 0 & 0 & 0 & 0 & 0 & 0 & 0 & 1 & 0 & 0 & 1 & 0 & 0 & 0 & 0 & 1 \\
\hline$v_{13}$ & 0 & 0 & 0 & 0 & 0 & 0 & 0 & 0 & 1 & 0 & 0 & 0 & 0 & 0 & 0 & 0 \\
\hline$v_{14}$ & 0 & 0 & 0 & 0 & 0 & 0 & 0 & 0 & 0 & 1 & 0 & 0 & 1 & 0 & 1 & 0 \\
\hline$v_{15}$ & 0 & 0 & 0 & 0 & 0 & 0 & 0 & 0 & 0 & 0 & 1 & 0 & 0 & 0 & 0 & 1 \\
\hline$v_{16}$ & & 0 & 0 & 0 & 0 & 0 & 0 & 0 & 0 & 0 & 0 & 0 & 0 & 0 & 1 & 0 \\
\hline
\end{tabular}

The Split adjacent matrix $a_{i j}$ is:

Since there exists a non-zero row in $a_{i j}$ and $v_{16}$ contains 1 in $v_{15}^{\text {th }}$ column $=v_{p}, \gamma_{s}=|C|+1=5$ 
Theorem 4.1. For any grid graph $G_{m, n}$,

$$
\gamma_{s}\left(G_{m, n}\right)= \begin{cases}\gamma\left(G_{m, n}\right) & \text { if }\left\langle V\left(G_{m, n}\right)-\gamma\left(G_{m, n}\right)\right\rangle \text { is disconnected } \\ \gamma\left(G_{m, n}\right)+1 & \text { otherwise. }\end{cases}
$$

Proof. Let $D$ be the $\gamma$-set of $G$.

Case 1: if $\langle V(G)-D\rangle$ is disconnected, the result follows from the definition of split dominating set.

Case 2: if $\langle V(G)-D\rangle$ is connected, then there exists atleast one vertex say $v_{i}$ in $D$ which is of degree 3 and $v_{i} \in N\left(v_{j}\right), v_{j}$ is of degree 2. Then $D \cup v_{k}, v_{k} \in N\left(v_{j}\right) \neq v_{i}$. Since $v_{j}$ is of degree 2, $D \cup v_{k}$ is disconnected. Hence $\gamma_{s}\left(G_{m, n}\right)=\gamma\left(G_{m, n}\right)+1$.

\section{Exact values of $\gamma_{s}\left(G_{m, n}\right), m \leq n$.}

The exact values of $\gamma_{s}\left(G_{m, n}\right)$ are obtained by using Theorem 4.1 and [1, 4]

$$
\begin{aligned}
& \gamma_{s}\left(G_{1, n}\right)=\left\lfloor\frac{n+2}{2}\right\rfloor \\
& \gamma_{s}\left(G_{2, n}\right)= \begin{cases}\left\lfloor\frac{n+2}{2}\right\rfloor & n \text { is even or } n=3 \\
\left\lfloor\frac{n+2}{2}\right\rfloor+1 & \text { otherwise. }\end{cases} \\
& \gamma_{s}\left(G_{3, n}\right)= \begin{cases}\left\lfloor\frac{3 n+4}{4}\right\rfloor & n \equiv 0(\bmod 4) \text { orn } n=3 \\
\left\lfloor\frac{3 n+4}{4}\right\rfloor+1 & \text { otherwise. }\end{cases} \\
& \gamma_{s}\left(G_{4, n}\right)=n+1 \text {. } \\
& \gamma_{s}\left(G_{5, n}\right)= \begin{cases}\frac{6 n+13}{5} & n=5 p+2, p \geq 2 \\
\left\lceil\frac{6 n+8}{5}\right\rceil & \text { otherwise. }\end{cases} \\
& \gamma_{s}\left(G_{6, n}\right)= \begin{cases}\left\lceil\frac{10 n+10}{7}\right\rceil & n \equiv 1(\bmod 7) \\
\frac{10 n+19}{7} & n=7 p+3, p \geq 1 \\
\left\lceil\frac{10 n+12}{7}\right\rceil & \text { otherwise }\end{cases} \\
& \gamma_{s}\left(G_{7, n}\right)= \begin{cases}\frac{5 n+6}{3} & n=3 p, p \geq 3 \\
\left\lceil\frac{5 n+3}{3}\right\rceil & \text { otherwise. }\end{cases} \\
& \gamma_{s}\left(G_{8, n}\right)= \begin{cases}\frac{15 n+22}{8} & n=8 p+6, p \geq 1 \\
\left\lceil\frac{15 n+14}{8}\right\rceil & \text { otherwise. }\end{cases} \\
& \gamma_{s}\left(G_{9, n}\right)= \begin{cases}\frac{23 n+31}{11} & n=11 p+2, p \geq 1 \\
\left\lceil\frac{23 n+20}{11}\right\rceil & \text { otherwise. }\end{cases} \\
& \gamma_{s}\left(G_{10, n}\right)= \begin{cases}\left\lceil\frac{30 n+37}{13}\right\rceil & n \neq 13,16, n \equiv 0,3(\bmod 13), n=13 p+7, p \geq 1 \\
\left\lceil\frac{30 n+24}{13}\right\rceil & \text { otherwise. }\end{cases}
\end{aligned}
$$




$$
\begin{aligned}
& \gamma_{s}\left(G_{11, n}\right)= \begin{cases}\left\lceil\frac{38 n+21}{15}\right\rceil & n=11,20,22 \\
\frac{38 n+51}{15} & n=15 p+3, p \geq 3 \\
\left\lceil\frac{38 n+36}{15}\right\rceil & \text { otherwise }\end{cases} \\
& \gamma_{s}\left(G_{12, n}\right)= \begin{cases}\frac{80 n+95}{29} & n=29 p-3, p \geq 1 \\
\left\lceil\frac{80 n+66}{29}\right\rceil & \text { otherwise. }\end{cases} \\
& \gamma_{s}\left(G_{13, n}\right)= \begin{cases}\left\lceil\frac{98 n+111}{33}\right\rceil & n \equiv 14,15,17,20(\bmod 33), n=33 p+12, p \geq 1 \\
\left\lceil\frac{98 n+78}{33}\right\rceil & \text { otherwise. }\end{cases} \\
& \gamma_{s}\left(G_{14, n}\right)= \begin{cases}\left\lceil\frac{35 n+40}{11}\right\rceil & n \equiv 18(\bmod 22), n=11 p+13, p \geq 1 \\
\left\lceil\frac{35 n+29}{11}\right\rceil & \text { otherwise. }\end{cases} \\
& \gamma_{s}\left(G_{15, n}\right)= \begin{cases}\left\lceil\frac{44 n+27}{13}\right\rceil+1 & n \equiv 5(\bmod 26) \\
\frac{44 n+53}{13} & n=13 p+5, p \geq 1 \\
\left\lceil\frac{44 n+40}{13}\right\rceil & \text { otherwise. }\end{cases} \\
& \gamma_{s}\left(G_{16, n}\right)= \begin{cases}\frac{18 n+21}{5} & n=5 p+13, p \geq 1 \\
\left\lceil\frac{18 n+36}{5}\right\rceil-4 & \text { otherwise. }\end{cases} \\
& \gamma_{s}\left(G_{17, n}\right)= \begin{cases}\frac{19 n+23}{5} & n=5 p+13, p \geq 1 \\
\left\lceil\frac{19 n+38}{5}\right\rceil-4 & \text { otherwise. }\end{cases} \\
& \gamma_{s}\left(G_{18, n}\right)=4 n+5 \text {. } \\
& \gamma_{s}\left(G_{19, n}\right)= \begin{cases}\frac{21 n+27}{5} & n=5 p+13, p \geq 2 \\
\left\lceil\frac{21 n+42}{5}\right\rceil-4 & \text { otherwise. }\end{cases} \\
& \gamma_{s}\left(G_{20, n}\right)= \begin{cases}\frac{22 n+29}{5} & n=5 p+13, p \geq 2 \\
\left\lceil\frac{22 n+44}{5}\right\rceil-4 & \text { otherwise. }\end{cases} \\
& \gamma_{s}\left(G_{21, n}\right)= \begin{cases}\frac{23 n+31}{5} & n=5 p+13, p \geq 2 \\
\left\lceil\frac{23 n+46}{5}\right\rceil-4 & \text { otherwise. }\end{cases} \\
& \gamma_{s}\left(G_{22, n}\right)= \begin{cases}\frac{24 n+33}{5} & n=5 p+13, p \geq 2 \\
\left\lceil\frac{24 n+48}{5}\right\rceil-4 & \text { otherwise. }\end{cases} \\
& \gamma_{s}\left(G_{23, n}\right)=5 n+7 \text {. } \\
& \gamma_{s}\left(G_{24, n}\right)= \begin{cases}\frac{26 n+37}{5} & n=5 p+13, p \geq 3 \\
\left\lceil\frac{26 n+52}{5}\right\rceil-4 & \text { otherwise. }\end{cases}
\end{aligned}
$$


Table 1. Split domination numbers $\gamma_{s}\left(G_{m, n}\right), m, n \leq 25$

\begin{tabular}{|c|c|c|c|c|c|c|c|c|c|c|c|c|c|c|c|c|}
\hline$n / n$ & 12 & 3 & & & & & & & & & I & 2 & 21 & 222 & 23 & $24 \quad 25$ \\
\hline 1 & - - & - & - & - & - & - & $-\quad-$ & $-\quad-$ & $-\quad-\quad-$ & - & - & - & - & - & - & - \\
\hline 2 & -2 & & & & & & & & & & & & & & & \\
\hline 3 & 12 & 3 & & & & & & & & & & & & & & \\
\hline 4 & 23 & 4 & 5 & & & & & & & & & & & & & \\
\hline 5 & 24 & 5 & 6 & 8 & & & & & & & & & & & & \\
\hline 6 & 24 & 6 & 7 & 911 & & & & & & & & & & & & \\
\hline 7 & 35 & 7 & 8 & 1012 & 213 & & & & & & & & & & & \\
\hline 8 & 35 & 7 & 91 & 1213 & 315 & 17 & & & & & & & & & & \\
\hline 9 & 36 & 81 & 10 & 1315 & 517 & 19 & 21 & & & & & & & & & \\
\hline 10 & $4 \quad 6$ & 91 & 111 & 1417 & 718 & & 2325 & & & & & & & & & \\
\hline 112 & 47 & 101 & 12 & 1518 & 820 & 23 & 2528 & 830 & & & & & & & & \\
\hline 12 & $47 \quad 1$ & 101 & 13 & 1715 & 922 & 25 & 2730 & 03336 & & & & & & & & \\
\hline 135 & 58 & 111 & 14 & 1821 & 123 & 27 & 3032 & 2363941 & & & & & & & & \\
\hline 145 & 58 & 121 & 15 & 1922 & 225 & 29 & 3235 & 5384145 & 548 & & & & & & & \\
\hline 155 & 59 & 131 & 162 & $202 ?$ & 327 & 30 & 3437 & 7414448 & 85154 & & & & & & & \\
\hline 16 & 69 & 131 & 172 & 2125 & 528 & 32 & 3639 & 9434750 & 0545861 & & & & & & & \\
\hline 17 ( & 6101 & 141 & 182 & 2327 & 730 & 34 & 3842 & 2465054 & 457616569 & & & & & & & \\
\hline 18 & 6101 & 151 & 192 & 2428 & 832 & 36 & 4044 & 4485256 & 661656973 & 77 & & & & & & \\
\hline $19 ?$ & 7111 & 162 & 202 & 2525 & 933 & 38 & 4246 & 6515559 & 964687276 & 81 & 85 & & & & & \\
\hline 207 & 7111 & 162 & 212 & 2631 & 135 & 40 & 4449 & 9535863 & 367717680 & 85 & 89 & 93 & & & & \\
\hline 217 & 7121 & 172 & 222 & 2732 & 237 & 42 & 4651 & 1566165 & 570757984 & 89 & 93 & 98 & 102 & & & \\
\hline 228 & 8121 & 182 & 232 & $293 ?$ & 338 & 44 & 4853 & 3586368 & 873788388 & 93 & 97 & 102 & & 112 & & \\
\hline 238 & 813 & 192 & 243 & 3035 & 540 & 45 & 5055 & 5616671 & 176818792 & 97 & 102 & 107 & & & 122 & \\
\hline 248 & 813 & 192 & 253 & 3137 & 742 & 47 & 5358 & 8646974 & 480859095 & 101 & 106 & 111 & & 1211 & & \\
\hline 25 & 9142 & 202 & $26 ?$ & 3238 & 843 & 49 & 5560 & 0667277 & 783889499 & 105 & 110 & 115 & & 1261 & & 37142 \\
\hline
\end{tabular}




\section{References}

[1] D. Goncalves, A. Pinlou, M. Rao and S. Thomasse, The domination of grid graph, SIAM J. Discrete Math. 25(3) (2011), 1443-1453.

[2] F. Harary, Graph Theory, Addison-wesley. reading Mass (1969).

[3] V. R. Kulli, B. Janikiram, The split domination number of a graph, Graph Theory Notes of New York Academy of Sciences. XXXII (1997),16-19.

[4] S. Alanko, A. Isopoussu, P. Ostergard, and V. Pettersson, Computing the domination number of grid graphs, Electron. J. Combin. 18 (2011), P141.

[5] T. W. Haynes, S. T. Hedetniemi, and P. J. Slater, Fundamentals of Domination in Graphs, Marcel Dekker, Inc. Newyork (1988). 